\title{
Multimodal imaging and genetic analysis of adult-onset best vitelliform macular dystrophy in Chinese patients
}

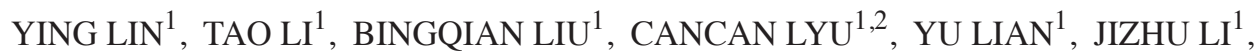 \\ YING HUANG ${ }^{1}$, HAICHUN LI ${ }^{1}$, QINGXIU WU ${ }^{1}$, CHENJIN JIN ${ }^{1}$ and LIN LU ${ }^{1}$ \\ ${ }^{1}$ State Key Laboratory of Ophthalmology, Zhongshan Ophthalmic Center, Sun Yat-sen University, Guangzhou, \\ Guangdong 510000, P.R. China; ${ }^{2}$ Department of Pharmacology, University of Iowa, Iowa City, IA 52242, USA
}

Received January 7, 2021; Accepted June 16, 2021

DOI: $10.3892 /$ etm.2021.10466

\begin{abstract}
Compared to juvenile-onset best vitelliform macular dystrophy (BVMD), adult-onset BVMD is not well characterized and lacks strict diagnostic criteria. The present study aimed to evaluate the clinical and genetic characteristics of four advanced-age Chinese patients with adult-onset BVMD by combining multimodal imaging and genetic analysis. The four patients (all older than 50 years) were diagnosed with adult-onset BVMD at Zhongshan Ophthalmic Center (Guangzhou, China). Comprehensive ophthalmic examinations were performed, including analyses of best-corrected visual acuity, intraocular pressure, slit-lamp examination, fundus photography, optical coherence tomography, fundus fluorescein angiography and electrooculography. Genomic DNA was extracted from leukocytes isolated from peripheral blood obtained from these patients, their family members and 200 unrelated subjects from the same population. A total of 11 exons of the bestrophin-1 (BEST1) gene were amplified using PCR and sequenced. All of the four patients presented with lesions in the macular area. The patients were diagnosed with adult-onset BVMD based on multimodal imaging and genetic analysis. A total of four recurrent mutations, namely c.763C $>\mathrm{T}$ (p.Arg255Trp, p.R255W) in exon 7, c.584C>T (p.Ala195Val, p.A195V) in exon 5, c.910_912del GAT (p.304delAsp, p.D304del) in exon 8 and c.310G >C (p.Asp104His, p.D104H) in exon 4 of BEST1, were identified. Sorting intolerant from tolerant predicted that the amino acid substitutions p.R255W, p.A195V and p.D104H in the BEST1 protein were causing the damage. Combining multimodal imaging and genetic analysis was helpful in confirming the diagnosis of patients with
\end{abstract}

Correspondence to: Professor Chenjin Jin or Professor Lin Lu, State Key Laboratory of Ophthalmology, Zhongshan Ophthalmic Center, Sun Yat-Sen University, 7 Jinsui Road, Guangzhou, Guangdong 510000, P.R. China

E-mail: jinchj@126.com

E-mail: lulin@gzzoc.com

Key words: multi-modal imaging, best vitelliform macular dystrophy, adult-onset, BEST1, mutation adult-onset BVMD. These results maybe valuable for clinical and genetic counseling and for the development of therapeutic interventions for patients with BVMD.

\section{Introduction}

Best vitelliform macular dystrophy (BVMD) is a hereditary retinal disease characterized by the accumulation of lipofuscin in the central macula of both eyes (1-3). Adult-onset BVMD was first described by Gass (4) more than four decades ago and is usually diagnosed after the age of 40 years $(1,4-6)$. Compared to juvenile-onset BVMD, adult-onset BVMD is associated with a wider spectrum of fundus abnormalities. The descriptions of the first nine cases by Gass (4) reflected the difficulty in differentiating adult-onset BVMD from the full spectrum of age-related macular degeneration (AMD) $(4,5,7)$. Thus, the lack of strict diagnostic criteria for adult-onset BVMD is challenging for clinicians and researchers.

Overall, eyes affected by VMD exhibit various patterns of progressive retinal pigment epithelium (RPE) alterations involving the macula, making it difficult to obtain a correct diagnosis according to the features of the imaging results. Peripherin-2, bestrophin-1 (BEST1), inter photoreceptor matrix proteoglycan-1(IMPG1) and IMPG2 are the genes detected in adult-onset BVMD $(5,8)$. These genes may be used as markers for the diagnosis of adult-onset BVMD if it manifests with adulthood macular degeneration, a variable degree of electrooculography (EOG) suppression. The present study aimed to address the clinical, histological, genetic, imaging and functional characteristics of adult-onset BVMD and further aimed to provide a comprehensive overview of the current understanding of adult-onset BVMD and its putative causes.

To this end, multimodal imaging and genetic analysis were combined to determine the clinical manifestations and genetic characteristics of four advanced-age Chinese patients with adult-onset BVMD.

\section{Patients and methods}

Study subjects and clinical examinations. All experimental protocols and methods were approved by the Ethics Committee of Zhongshan Ophthalmic Center of Sun Yat-sen University 
(Guangzhou, China). Written informed consent was obtained from all of the participants who were treated according to the Declaration of Helsinki.

A total of four older patients $>50$ years with suspected adult-onset BVMD were encountered at the Zhongshan Ophthalmic Center (Guangzhou, China; between March 2017 and March 2021). Ophthalmic examinations were performed as follows: Visual acuity was examined using an Early Treatment Diabetic Retinopathy Study chart (Precision Vision). Optical coherence tomography (OCT) was performed using a Cirrus HD-OCT (Carl Zeiss Meditec). Three patients (Patients no. 1, 2 and 4) underwent OCT. Fundus photography and fundus fluorescein angiography (FFA) imaging were performed using a Heidelberg Retina Angiograph (Heidelberg Engineering). EOG was recorded in all patients and these tests were performed in conformity with the guidelines of the International Society for Clinical Electrophysiology of Vision standards (9). Physical examination was performed to exclude any systemic diseases.

Sample collection and mutation screening. Venous blood samples were collected from these four patients, their family members and 200 subjects without BVMD from the same region (Guangdong, China). The normal control presented in the Results section is a 50-year-old male, recruited by volunteering in November 2017. Genomic DNA was extracted from peripheral blood leukocytes using standard protocols. Exons of the BEST1 gene were amplified by PCR using primers (Table I) as previously described $(8,10)$. PCR was performed in 50- $\mu \mathrm{l}$ reactions. Amplification included a single 5-min step at $94^{\circ} \mathrm{C}$, followed by 40 cycles of $94^{\circ} \mathrm{C}$ for $45 \mathrm{sec}, 58-61^{\circ} \mathrm{C}$ for $45 \mathrm{sec}$ and $72^{\circ} \mathrm{C}$ for $45 \mathrm{sec}$, and a final 10 -min step at $72^{\circ} \mathrm{C}$. The PCR products were sequenced in both directions using an ABI3730 Automated Sequencer (Thermo Fisher Scientific, Inc.). The sequencing results were analyzed using Seqman (version 2.3; Technelysium Pty., Ltd.) and compared with the reference sequences in the database of the National Center for Biotechnology Information (NCBI; NC_000011.10).

To analyze the effect of missense variants, polymorphism phenotyping (PolyPhen; http://genetics.bwh.harvard. edu/pph2/) and sorting intolerant from tolerant (SIFT; http://sift.jcvi.org/) were used to predict the possible impact of an amino acid substitution on the structure and function of the protein using straight forward physical and comparative considerations. Variants were considered to be pathogenic when at least one of the two programs predicted a deleterious effect of amino acid substitution on protein structure and function. The Human Gene Mutation Database (http://www.hgmd. org/) was used to screen for mutations reported in published studies. HomoloGene (https://www.ncbi.nlm.nih.gov/homologene) was used to check whether the mutated amino acid residues were conserved across different species.

\section{Results}

Clinical findings. All patients were from southern China. A 62-year-old female patient (Patient no. 1; Fig. 1) had no known familial history of ocular diseases. Her best-corrected visual acuity (BCVA) was 20/100 in both eyes, which was not possible to be corrected. The cornea was transparent and there were certain opacities in the lens. Fundus examination indicated that certain pigments in the macular area and the fovea reflex were negative (Fig. 1A and D) compared to anormal control subject (Fig. 1H). OCT scans revealed that the foveal region was abnormally thick in both eyes due to neuroretinal detachment from the retinal pigment epithelium (RPE; Fig. 1B and E), which was likely triggered by the abnormal accumulation of subretinal fluid compared to the normal control (Fig. 1I). FFA indicated pooling of fluorescein dye in the cystoid spaces in the late phase of fluorescein angiography, which may have been caused by the RPE defect (Fig. 1C and F). The Arden ratio (light peak/dark trough) was markedly reduced to $<1.6$. Chronic central serous chorioretinopathy (CSC) was initially diagnosed and the patient was treated with photodynamic therapy (PDT) with no clinical response.

A 50-year-old male patient's (Patient no. 2; Fig. 2) BVCA was 20/80 in both eyes. The cornea and lens were transparent. Fundus photographs revealed round lesions in the macular area (Fig. 2A and D). OCT indicated serous retinal detachment (Fig. 2B and E). A fluorescein angiogram displayed hyperfluorescence in the macular area (Fig. $2 \mathrm{C}$ and F). The Arden ratio was reduced to $<1.6$. Chronic CSC was initially diagnosed and treated with PDT with no improvement in vision.

A 60-year-old male patient's (patient no. 3; Fig. 3) BCVA was $20 / 200$ in the right eye and counting fingers (CF) at $30 \mathrm{~cm}$ $(\mathrm{CF} / 30 \mathrm{~cm})$ in the left eye. The cornea was transparent and there were certain opacities in the lens. Fundus examination indicated a yolk-like lesion in the macula of the right eye and an atrophic lesion in the left eye (Fig. 3A and C). FFA revealed a small amount of hyperfluorescence in the right eye, which may have been caused by the RPE defect, and certain hyperfluorescence in the macular area of the left eye corresponding to the region containing the atrophic lesion detected on color fundus photography (Fig. 3B and D). The Arden ratio was reduced to $<1.8$.

A 78-year-old male patient's (Patient no. 4; Fig. 4) BVCA was $\mathrm{CF} / 20 \mathrm{~cm}$ in the right eye and $\mathrm{CF} / 30 \mathrm{~cm}$ in the left eye. The cornea was transparent. Severe opacities in the left lens and an intraocular lens in the right eye with certain opacity in the posterior capsule were observed and the fundus photographs were therefore not very clear. Fundus photography indicated round lesions in the macular area and a venous loop in the inferior part of the optic nerve head (Fig. 4A and D). OCT scans revealed that serous retinal detachments with elongated photoreceptor outer segments were correlated with the size of the yellowish elevated lesion (Fig. 4B and E). A fluorescein angiogram revealed a region of blocked fluorescence in the foveal center surrounded by a transmission defect in the FFA (Fig. 4C and F). The Arden ratio was severely reduced to $<1.4$. Chronic CSC was diagnosed at first and the patient was treated with PDT with no improvement in vision.

One characteristic that was similar in all of the four patients was that it was difficult to obtain an accurate diagnosis due to the onset age of the disease and its atypical clinical manifestations. Hence, the genetic analysis results have an important role in adult-onset BVMD.

Mutation screening and bioinformatics analysis. In Patient no. 1, a heterozygous mutation, c. $763 \mathrm{C}>\mathrm{T}$ (p. Arg255Trp, p.R255W), was identified in exon 7 of BEST1 (Fig. 1G). In 
Table I. Primers used for the amplification of the exons of bestrophin-1and product sizes.

\begin{tabular}{cllrc}
\hline Exon & \multicolumn{1}{c}{ Forward (5'-3') } & \multicolumn{1}{c}{ Reverse (5'-3') } & $\begin{array}{c}\text { Product } \\
\text { size, bp }\end{array}$ & $\begin{array}{c}\text { Annealing } \\
\text { temperature, }{ }^{\circ} \mathrm{C}\end{array}$ \\
\hline 2 & AGTCTCAGCCATCTCCTCGC & TGGCCTGTCTGGAGCCTG & 212 & 61 \\
3 & GGGACAGTCTCAGCCATCTC & CAGCTCCTCGTGATCCTCC & 238 & 58 \\
4 & AGAAAGCTGGAGGAGCCG & GCGGCAGCCCTGTCTGTAC & 1408 & 59 \\
5 & GGGGCAGGTGGTGTTCAGA & GGCAGCCTCACCAGCCTAG & 150 & 59 \\
6 & GGGCAGGTGGTGTTCAGA & CCTTGGTCCTTCTAGCCTCAG & 181 & 59 \\
7 & CATCCTGATTTCAGGGTTCC & CTCTGGCCATGCCTCCAG & 257 & 59 \\
8 & AGCTGAGGTTTAAAGGGGGA & TCTCTTTGGGTCCACTTTGG & 215 & 59 \\
9 & ACATACAAGGTCCTGCCTGG & GCATTAACTAGTGCTATTCTAAGTTCC & 298 & 59 \\
10 A & GGTGTTGGTCCTTTGTCCAC & CTCTGGCATATCCGTCAGGT & 591 & 59 \\
$10 B$ & CTTCAAGTCTGCCCCACTGT & TAGGCTCAGAGCAAGGGAAG & 457 & 59 \\
11 & CATTTTGGTATTTGAAATGAAGG & CCATTTGATTCAGGCTGTTG & 216 & 59 \\
\hline
\end{tabular}

Patient no. 2, the mutation c.584C >T (p.Ala195Val, p.A195V) was identified in exon 5 of BEST1 (Fig. 2G). In Patient no. 3 , the mutation c.910_912del GAT (p.Asp304del, p.D304del) was identified in exon 8 of BEST1 (Fig. 3E). In Patient no. 4, the mutation c.310G >C (p.Asp104His, p.D104H) was identified in exon 4 of the BEST1 gene (Fig. 4G). No equivalent mutations were detected in the family members of these subjects or the control subjects. SIFT predicted that the amino acid substitutions Arg255Trp, Ala195Val, and Asp104His in the BEST1 protein were damaging to its function (Fig. 5A-C). PolyPhen was not able to predict the effects of these four mutations.

\section{Discussion}

Adult-onset BVMD is one of the most prevalent forms of macular degeneration and was first described by Gass in 1974 (4). Compared to juvenile-onset BVMD, adult-onset BVMD is less characterized and lacks strict diagnostic criteria. It was suggested that adult-onset BVMD typically occurs between 30 and 50 years of age (5). The patients in the present study were older than 50 years, similar to those in previous studies (7), indicating that adult-onset BVMD cannot be excluded in older patients with macular lesions.

The clinical symptoms of adult-onset BVMD are highly variable, but patients tend to remain asymptomatic until the 5 th decade or may even remain asymptomatic throughout life (11). Atypical presentations, such as multifocal vitelliform lesions and the occurrence of choroidal neovascularization (CNV) in various stages of the disease, may confound the diagnosis $(8,12,13)$. In previous studies, the majority of cases had a negative family history of the disease (5), similar to the four cases in the present study. Furthermore, adult-onset BVMD-like lesions have been suggested to be associated with several additional phenotypes and pathologies, such as AMD (7) and chronic CSC $(5,14)$. A study by Spaide et al $(15)$ reported vitelliform lesions in three patients who were diagnosed with typical CSC. In this study, three patients were misdiagnosed with CSC, as the vitelliform lesions caused mechanical separation between the photoreceptors and RPE.
Therefore, for patients with adult-onset BVMD, combining multimodal imaging and genetic examination is helpful for confirming the diagnosis when other causes of macular atrophy may be excluded (7,16-18).

BVMD is highly causally associated with mutations in the gene BEST1 (19) which encodes BEST1. Human BEST1, identified in 1998, is a calcium-activated chloride channel in the RPE (11).

In addition to evaluating clinical and genetic manifestations of BVMD, the present study sought to investigate the correlations between genotypes and phenotypes in order to provide additional criteria for this disease for ophthalmologists to avoid an incorrect diagnosis. A study performed in Japan identified one patient with heterozygous variants of V239VfsX2/p.R255W, who presented mainly with macular edema and low amounts of subretinal fluid (Table II) (20) unlike what was detected in Patient no. 1 of the present study. First, the phenotypes of compound heterozygous mutations and those associated with this one mutation maybe different. Furthermore, the patients in the Japanese study had juvenile-onset BVMD, which may differ from adult-onset BVMD, which was the focus of the present study. Furthermore, the p.R255W mutation may not be related to subretinal fluid, which is reasonable because BVMD involves 4-5 stages. Wong et al (21) reported another patient, an 8-year-old male with BVMD and CNV (Table II). Tian et al (22) and another study (23) from the glaucoma division of our hospital also identified that certain patients with p.R255W had angle-closure glaucoma in addition to macular lesions (Table II). Thus, these results may confirm that addressing the role of p.R255Win BVMD is important in both juvenile-onset and adult-onset BVMD and may present with numerous clinical features, which makes it a hot topic in the field. According to the protein structure of BEST1, amino acid 255 is positioned at the margin of the cell membrane (19); p.R255W therefore deserves further research regarding the mechanism by which it contributes to BVMD (24).

The variant p.A195V had been previously reported in a 50-year-old Japanese male with adult-onset BVMD (Table II) (25), whose vision was much better than that of Patient no. 2 in the 
A

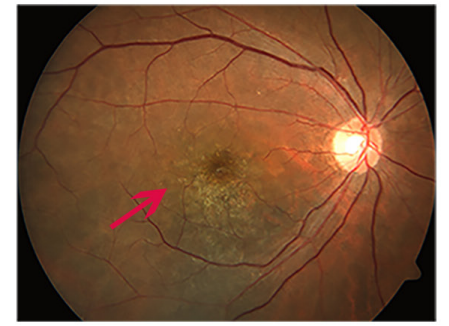

D

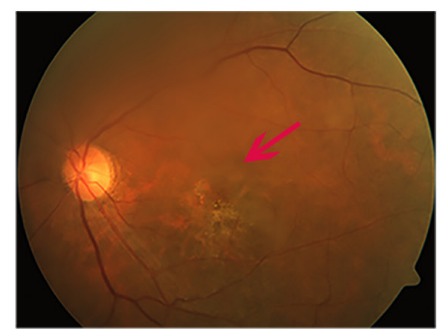

B
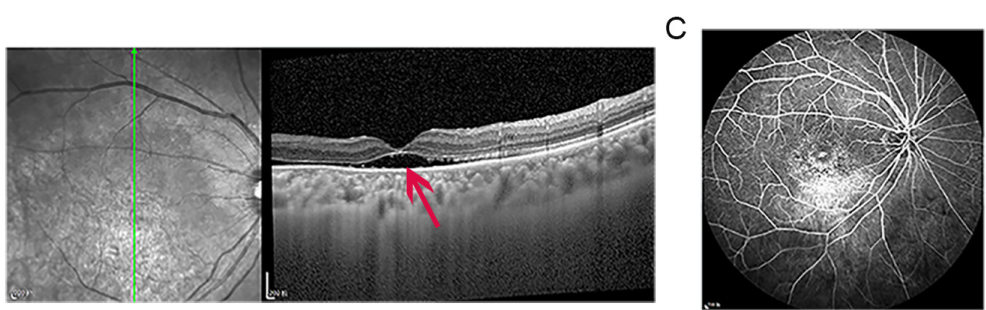

E

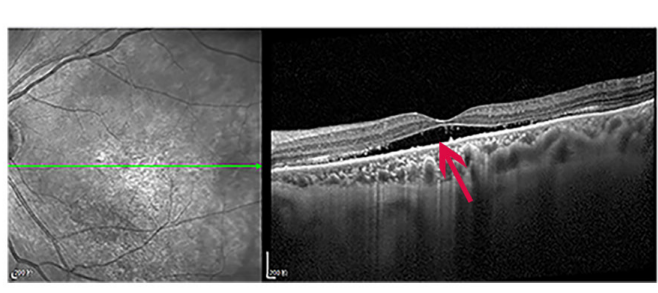

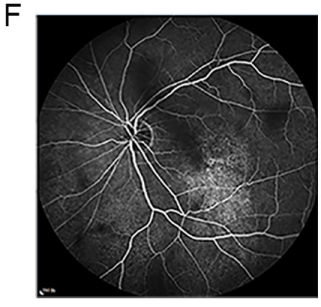

G

Wild

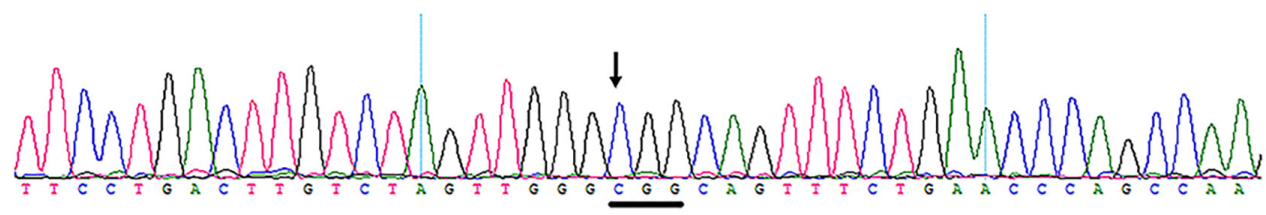

Mutant

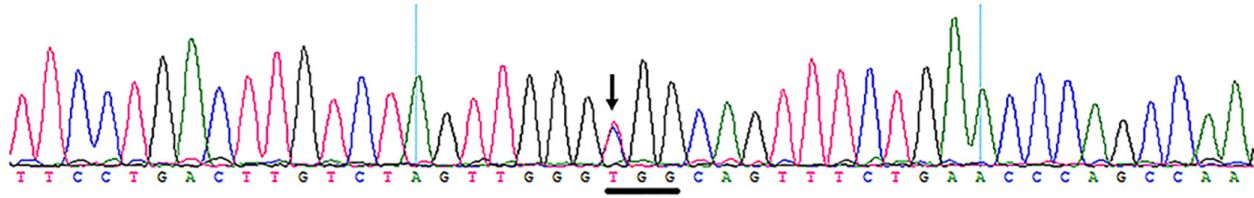

$\mathrm{H}$
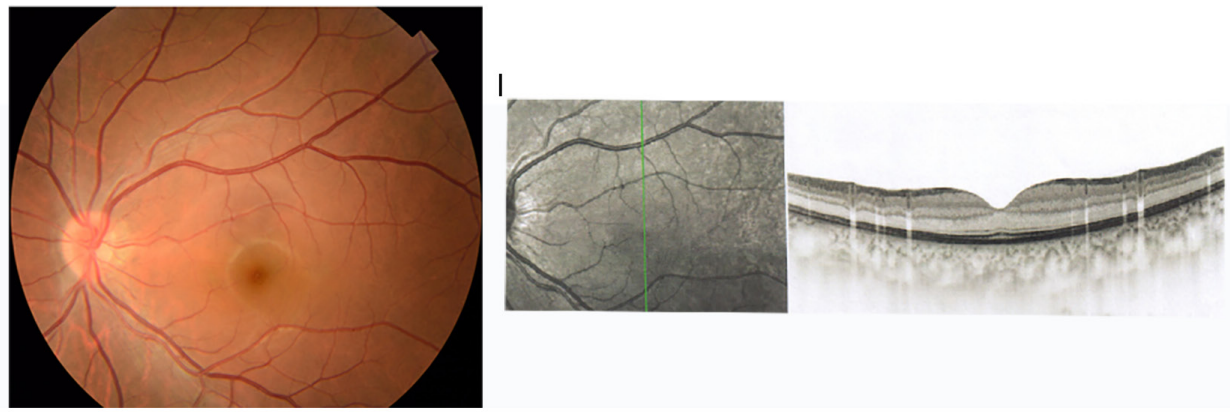

Figure 1. Clinical features and genetic results of Patient no. 1, a 62-year-old female. (A-C) Right eye and (D-F) left eye. (A and D) Fundus examination indicated that certain pigments in the macular area and the fovea reflex were negative (red arrows). (B and E) OCT scans revealed that the foveal regions of both eyes were abnormally thick due to neuroretinal detachment from the RPE, which was likely triggered by the abnormal accumulation of subretinal fluid (red arrows). (C and F) FFA indicates pooling of fluorescein dye in the cystoid spaces in the late phase of FFA, which may have been caused by the RPE defect. (G) In Patient no. 1, a heterozygous mutation, c.763C > T (p. Arg255Trp, p.R255W), was identified in exon 7 of the bestrophin-1gene. (H) Fundus photo of normal control (a 50-year-old male). (I) OCT scan of normal control (the same as in H). RPE, retinal pigment epithelium; OCT, optical coherence tomography; RPE, retinal pigment epithelium; FFA, fundus fluorescein angiography; wild, wild-type.

present study, although both patients were 50-year-old males. Lee et al (26) determined that 293T cells transfected with a p.A195V mutant produced significantly smaller currents than cells transfected with the wild-type gene. Tian et al (27) also reported a case of the compound mutation p.A195V/p.R255W in a young patient with good vision, which may indicate that the compound mutation is not as severe as the single mutation, similar to the results of a previous study (28). It may be suggested that the reason is that certain mutations increase $\mathrm{Cl}^{-}$currents but that others decrease $\mathrm{Cl}^{-}$currents.

To the best of our knowledge, as for Patient no. 3 of the present study, only one previous study by Katagiri et al (25) detected the c.910_912del GAT mutation in a 56-year-old Japanese male. Although c.910G >A (p.Asp304Asn) was identified by another investigator, no detailed description was provided (https://www.ncbi.nlm.nih.gov/clinvar/variation/496689/). These results indicated that Asp in this position is important for the function of the whole protein. According to the structure of the BEST1 protein $(8,29,30)$, the amino acids in positions 301-304 are all Asp and all mutations at these sites cause BVMD, which may indicate that the structure of several continuous residues of the same amino acid is easily damaged.

The c.310G $>C$ (p.Asp104His, p.D104H) mutation in exon 4 is also a recurrent mutation, as reported by Krämer et al (31) 
A
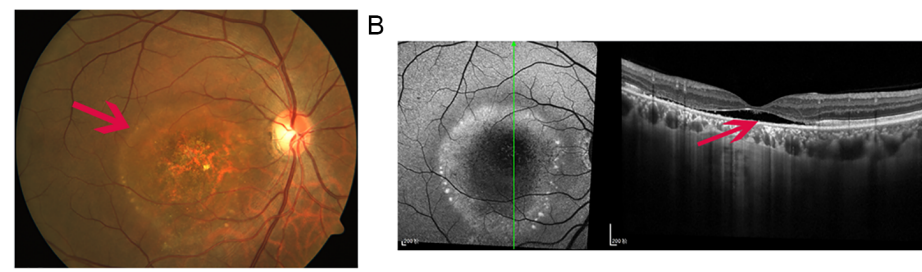

D
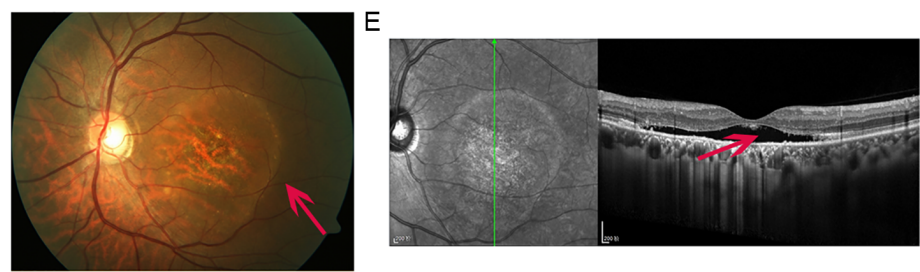

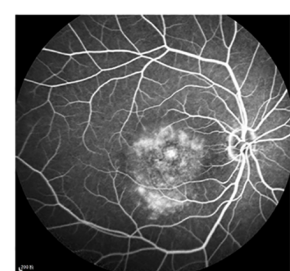

$\mathrm{F}$

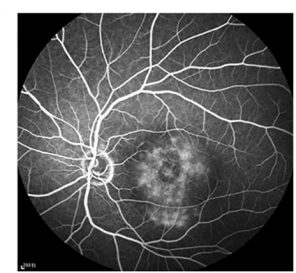

G

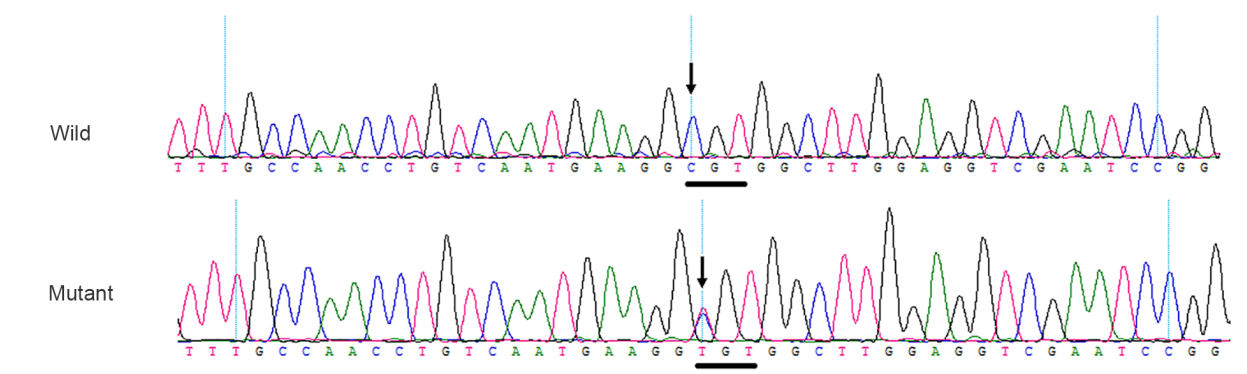

Figure 2. Clinical features and genetic results of Patient no. 2, a 50-year-old male. (A-C) Right eye and (D-F) left eye. (A and D) Fundus photograph indicates round lesions in the macular area (red arrows). (B and E) Optical coherence tomography scans reveal serous retinal detachments (red arrows). (C and F) Fluorescein angiogram indicating hyperfluorescence in the macular area. $(\mathrm{G})$ In Patient no. 2, c.584C $>$ T (p.Ala195Val, p.A195V) was identified in exon 5 of the bestrophin-1gene. Wild, wild-type.
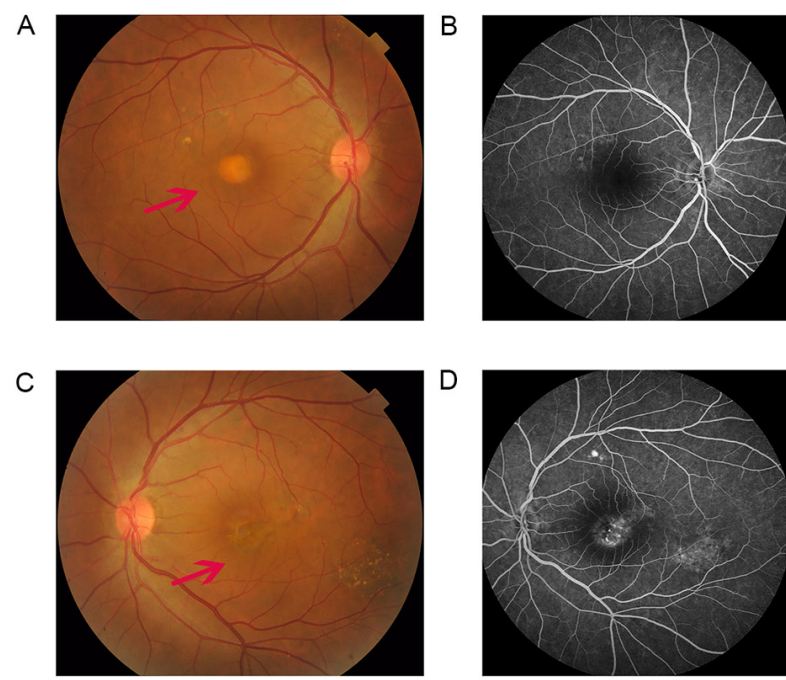

$\mathrm{E}$ Wild

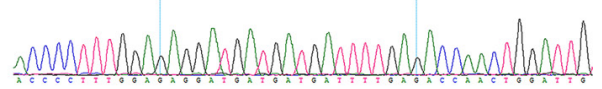

Mutant

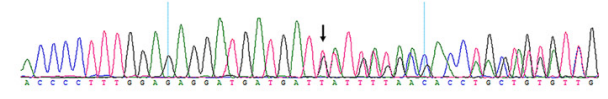

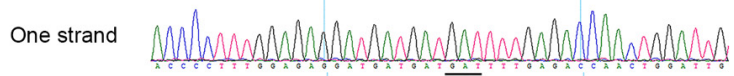

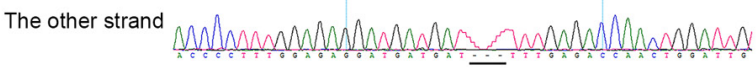

Figure 3. Clinical features and genetic results of Patient no. 3, a 60-year-old male. (A and B) Right eye and (C and D) left eye. (A and C) Fundus examination indicated a yolk-like lesion in the macula of the right eye and an atrophic lesion in the left eye (red arrows). (B and D) Fundus fluorescein angiography revealed a small amount of hyperfluorescence in the right eye, which may have been caused by the retinal pigment epithelium defect, and a certain amount of hyperfluorescence in the macular area of the left eye, corresponding to the region containing the atrophic lesion observed on color fundus photography. (E) In Patient no. 3, c.910_912del GAT (p.Asp304del, p.D304del) in exon 8 of the bestrophin-1gene was identified. Wild, wild-type.

(Table II). The Asp104 residue is highly conserved among other species, including nematodes and fruit flies. Finally, codon 104 has previously been indicated to be affected by a mutational change that altered the asparagine residue to a glutamic residue (Asp104Glu) (32). Taken together, these data indicate that Asp104 is likely to be the causative agent of BVMD.
Currently, no validated therapy is available for restoration of the normal contact between the photoreceptors and the RPE and/or for facilitating safe lesion absorption (5). Ergun et al (33) reported that PDT had no beneficial effect on the vision of patients with BVMD, suggesting that severe adverse effects were associated with this treatment. In the present study, Patients no. 1, 2 and 4 were treated 
A

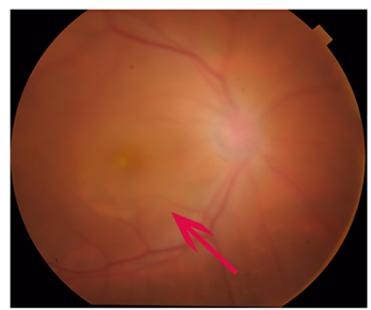

B

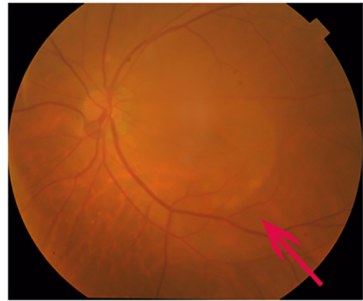

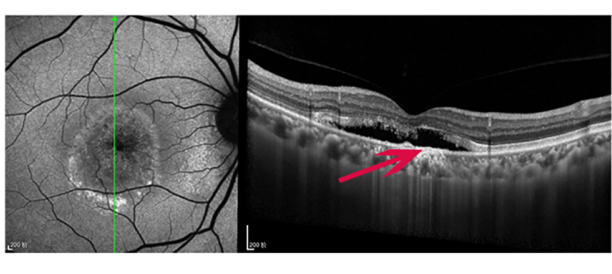

C
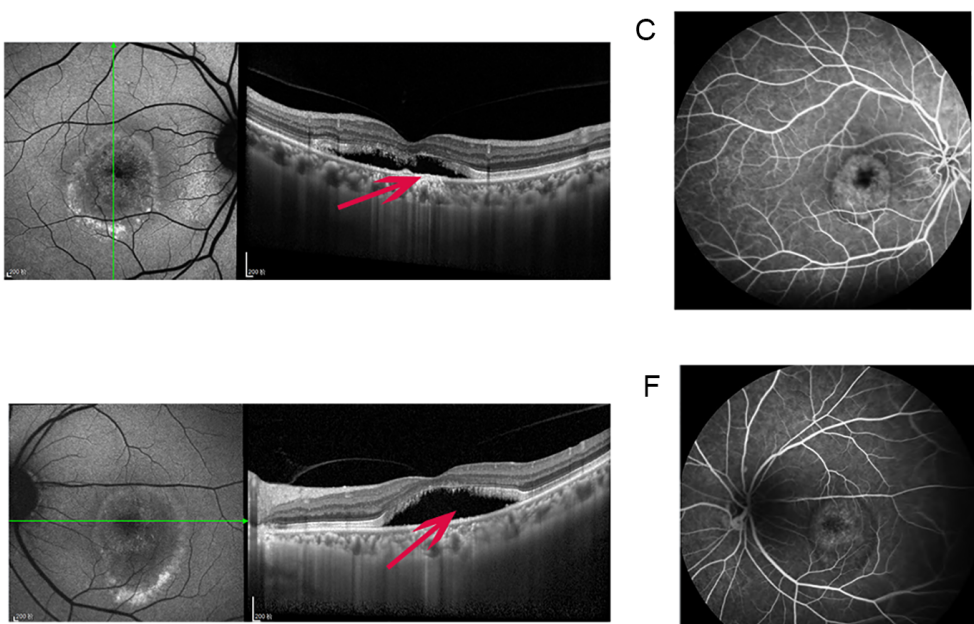

$\mathrm{F}$

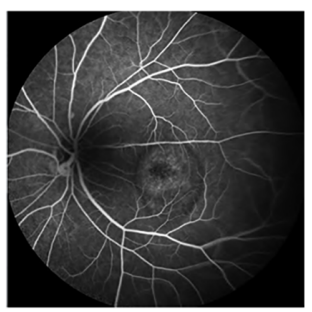

G

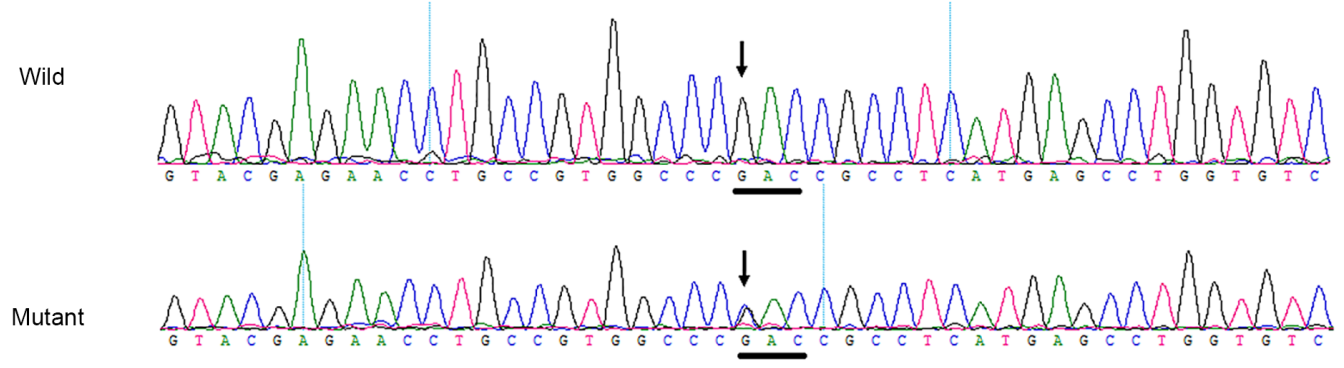

Figure 4. Clinical features and genetic results of Patient no. 4, 78-year-old male. (A-C) Right eye and (D-F) left eye. (A and D) Fundus photographs indicating round lesions in the macular area and a venous loop in the inferior optic nerve head (red arrows). (B and E) Optical coherence tomography scans reveal that serous retinal detachments with elongated photoreceptor outer segments are correlated with the size of the yellowish elevated lesion (red arrows). (C and F) Fundus fluorescein angiography indicating a region of blocked fluorescence in the foveal center surrounded by a transmission defect in the fundus. (G) In Patient no. 4, a c.310G>C (p.Asp104His, p.D104H) was identified in exon 4 of the bestrophin-1gene. Wild, wild-type.
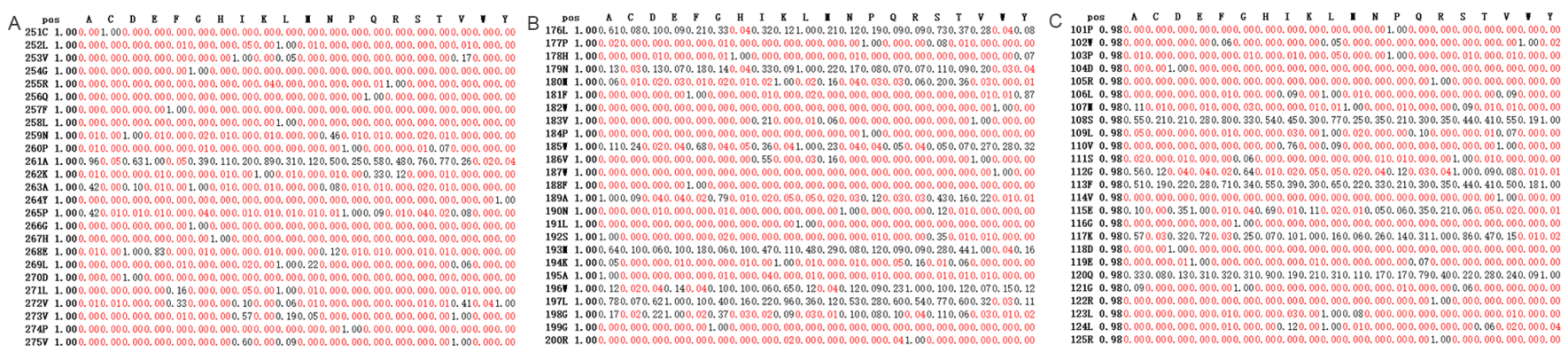

Figure 5. SIFT prediction. (A) SIFT predicted that the amino acid substitution Arg255Trp in the BEST1 protein is damaging. (B) The amino acid substitution Ala195Val in the BEST1 protein was predicted to be damaging by SIFT. (C) SIFT predicted that the amino acid substitution Asp104His in the BEST1 protein is damaging. Red numbers indicate the damaging and black numbers correspond to the not damaging amino acid substitutions. SIFT, sorting intolerant from tolerant; pos, position; BEST1, bestrophin-1.

with PDT without any favorable outcomes. The prognosis of adult-onset BVMD is poor, as visual impairment and legal blindness may occur, as was observed in the present study. Gene therapy is the most promising option for treating monogenic forms of BVMD prior to fluid-induced rod cell death (11,34).

Certain limitations of the present study should be noted. First, the sample size of the included subjects was limited because of the rarity of adult-onset BVMD. Furthermore, no longitudinal study was performed and therefore, it was not possible to assess the temporal trends of these changes during aging.

In summary, by combining multimodal imaging and genetic examination, adult-onset BVMD was confirmed in four patients. These results expand the mutation spectrum of BEST1and may be helpful in genetic and clinical counseling to correctly diagnose patients with adult-onset BVMD. In addition, the characterization of these patients with adult-onset BVMD in the present study provides a basis for future investigations of the mechanisms underlying 
Table II. Comparison of clinical features of the same mutation between the present study and other studies.

\begin{tabular}{|c|c|c|c|c|c|c|c|}
\hline Study (year) & Mutation & Region & Sex & $\begin{array}{c}\text { Age } \\
\text { (years) }\end{array}$ & Visual acuity & Fundus appearance & (Refs.) \\
\hline Present study & $\begin{array}{l}\text { p.R255W (heterozygous) } \\
\text { Patient no. } 1\end{array}$ & Asia (China) & $\mathrm{F}$ & 62 & OU: $20 / 100$ & $\begin{array}{l}\text { Pigmentation in the } \\
\text { macular area }\end{array}$ & - \\
\hline $\begin{array}{l}\text { Kubota et al, } \\
2016\end{array}$ & $\begin{array}{l}\text { V239VfsX2/p.R255W } \\
\text { (heterozygous) }\end{array}$ & Asia (Japan) & M & 25 & $\begin{array}{l}\text { OD: } 0.9 \\
\text { OS: } 0.3 \\
\text { (decimal) }\end{array}$ & $\begin{array}{l}\text { Cystoid macular } \\
\text { lesions and multiple } \\
\text { yellowish deposits }\end{array}$ & $(20)$ \\
\hline $\begin{array}{l}\text { Wong et al, } \\
2010\end{array}$ & p.R255W (heterozygous) & Asia (China) & M & 8 & $\begin{array}{l}\text { OD: } 20 / 200 \\
\text { OS: } 20 / 30\end{array}$ & $\begin{array}{l}\text { OD: CNV } \\
\text { OS: Vitelliruptive }\end{array}$ & $(21)$ \\
\hline $\begin{array}{l}\text { Tian et al, } \\
2017\end{array}$ & p.R255W (heterozygous) & Asia (China) & NS & NS & NS & $\begin{array}{l}\text { Diffuse yellowish } \\
\text { lesion in the macula; } \\
\text { the C/D ratio was } 0.8\end{array}$ & $(22)$ \\
\hline $\begin{array}{l}\text { Luo et al, } \\
2019\end{array}$ & $\begin{array}{l}\text { p.R255W (homozygous, } \\
\text { heterozygous) }\end{array}$ & Asia (China) & NS & NS & NS & $\begin{array}{l}\text { Angle-closure } \\
\text { glaucoma and } \\
\text { macular lesion }\end{array}$ & (23) \\
\hline Present study & $\begin{array}{l}\text { p.A195V (heterozygous) } \\
\text { Patient no. } 2\end{array}$ & Asia (China) & M & 50 & OU: $20 / 80$ & $\begin{array}{l}\text { Round lesions } \\
\text { in the macular area }\end{array}$ & - \\
\hline $\begin{array}{l}\text { Katagiri et al, } \\
2015\end{array}$ & p.A195V (heterozygous) & Asia (Japan) & M & 50 & $\begin{array}{c}\text { OD: } 1.2 \\
\text { OS: } 1.0 \\
\text { (decimal) }\end{array}$ & $\begin{array}{l}\text { OD: Vitelliruptive } \\
\text { stage } \\
\text { OS: Vitelliruptive stage }\end{array}$ & $(25)$ \\
\hline $\begin{array}{l}\text { Tian et al, } \\
2014\end{array}$ & $\begin{array}{l}\text { p.A195V/p.R255W } \\
\text { (heterozygous) }\end{array}$ & Asia (China) & NS & 25 & $\begin{array}{l}\text { OD: } 20 / 50 \\
\text { OS: } 20 / 40\end{array}$ & $\begin{array}{l}\text { OD: Pseudohypopyon } \\
\text { OS: Vitelliform lesion }\end{array}$ & $(27)$ \\
\hline Present study & $\begin{array}{l}\text { p.D304del (heterozygous) } \\
\text { Patient no. } 3\end{array}$ & Asia (China) & M & 60 & $\begin{array}{c}\text { OD: } 20 / 200 \\
\text { OS:CF/30 cm }\end{array}$ & $\begin{array}{l}\text { OD: Yolk-like lesion } \\
\text { in the macula } \\
\text { OS: Atrophic lesion }\end{array}$ & - \\
\hline $\begin{array}{l}\text { Katagiri et al, } \\
2015\end{array}$ & p.D304del (heterozygous) & Asia (Japan) & M & 56 & $\begin{array}{l}\text { OD:0.15 } \\
\text { OS: } 1.2 \\
\text { (decimal) }\end{array}$ & $\begin{array}{l}\text { OD: Atrophic stage } \\
\text { OS: Vitelliform stage }\end{array}$ & $(25)$ \\
\hline Present study & $\begin{array}{l}\text { p.D104H (heterozygous) } \\
\text { Patient no. } 4\end{array}$ & Asia (China) & M & 78 & $\begin{array}{l}\mathrm{CF} / 20 \mathrm{~cm} \mathrm{OD} \\
\mathrm{CF} / 30 \mathrm{~cm} \mathrm{OS}\end{array}$ & $\begin{array}{l}\text { Round lesions in the } \\
\text { macular area }\end{array}$ & - \\
\hline $\begin{array}{l}\text { Krämer et al, } \\
2003\end{array}$ & p.D104H (heterozygous) & Germany & & NS & NS & NS & $(31)$ \\
\hline
\end{tabular}

F, female; M, male; CNV, choroidal neovascularization; OU, both eyes; OD, right eye; OS, left eye; CF, counting fingers; NS, not specified.

the pathogenesis and for the development of therapeutic interventions.

\section{Acknowledgements}

Not applicable.

\section{Funding}

This study was supported by the National Natural Science Foundation of China (grant no. 82070972).

\section{Availability of data and materials}

All data generated or analyzed during this study are included in this published article.

\section{Authors' contributions}

YLin, TL, BL, CJ and LL analyzed and interpreted the patient data. CL, YLian, YH, QW, HL and JL examined the patients and performed PCR and gene sequence analysis. YLin, JL and HL interpreted the sequencing data, drafted the manuscript and revised it critically. YLin and LL confirm the authenticity of all the raw data. All authors read and approved the final manuscript.

\section{Ethics approval and consent to participate}

All experimental protocols were approved by the Ethics Committee of Zhongshan Ophthalmic Center (Guangzhou, China). Written informed consent was obtained from all subjects. 


\section{Patient consent for publication}

Written informed consent for publication was obtained from all participants.

\section{Competing interests}

The authors declare that they have no competing interests.

\section{References}

1. MacDonald IM and Lee T: Best vitelliform macular dystrophy. In: GeneReviews $((\mathrm{R}))$. Adam MP, Ardinger $\mathrm{HH}$ and Pagon RA (eds). University of Washington, Seattle, WA, 1993.

2. Katz MS, Walsh EK and Medow NB: Vitelliform macular dystrophy. JAMA Ophthalmol 132: 1098, 2014.

3. Tsang SH and Sharma T: Best vitelliform macular dystrophy. Adv Exp Med Biol 1085: 157-158, 2018.

4. Gass JD: A clinicopathologic study of a peculiar foveomacular dystrophy. Trans Am Ophthalmol Soc 72: 139-156, 1974.

5. Chowers I, Tiosano L, Audo I, Grunin M and Boon CJ: Adult-onset foveomacular vitelliform dystrophy: A fresh perspective. Prog Retin Eye Res 47: 64-85, 2015.

6. Jun I, Lee JS, Lee JH, Lee CS, Choi SI, Gee HY, Lee MG and Kim EK: Adult-onset vitelliform macular dystrophy caused by BEST1 p.Ile38Ser mutation is a mild form of best vitelliform macular dystrophy. Sci Rep 7: 9146, 2017.

7. Toto L, Borrelli E, Mastropasqua R, Di Antonio L, Mattei PA, Carpineto P and Mastropasqua L: Adult-onset foveomacular vitelliform dystrophy evaluated by means of optical coherence tomography angiography: A comparison with dry age-related macular degeneration and healthy eyes. Retina 38: 731-738, 2018.

8. Lin Y, Li T, Ma C, Gao H, Chen C, Zhu Y, Liu B, Lian Y, Huang Y, $\mathrm{Li} \mathrm{H}$, et al: Genetic variations in bestrophin-1 and associated clinical findings in two Chinese patients with juvenile-onset and adult-onset best vitelliform macular dystrophy. Mol Med Rep 17: 225-233, 2018.

9. Constable PA, Bach M, Frishman LJ, Jeffrey BG and Robson AG International Society for Clinical Electrophysiology of Vision: ISCEV standard for clinical electro-oculography (2017 update) Doc Ophthalmol 134: 1-9, 2017.

10. Lin Y, Li T, Gao H, Lian Y, Chen C, Zhu Y, Li Y, Liu B, Zhou W, Jiang H, et al: Bestrophin 1 gene analysis and associated clinical findings in a Chinese patient with best vitelliform macular dystrophy. Mol Med Rep 16: 4751-4755, 2017.

11. Yang T, Justus S, Li Y and Tsang SH: BEST1: The best target for gene and cell therapies. Mol Ther 23: 1805-1809, 2015.

12. Shahzad R and Siddiqui MA: Choroidal neovascularization secondary to best vitelliform macular dystrophy detected by optical coherence tomography angiography. J AAPOS 21: 68-70, 2017.

13. Stattin M,AhmedD, Glittenberg C,Krebs I and Ansari-Shahrezaei S: Optical coherence tomography angiography for the detection of secondary choroidal neovascularization in vitelliform macular dystrophy. Retin Cases Brief Rep 14: 49-52, 2020.

14. Giuffrè C, Miserocchi E, Modorati G, Carnevali A, Marchese A, Querques L, Querques G and Bandello F: Central serous chorioretinopathylike mimicking multifocal vitelliform macular dystrophy: An ocular side effect of mitogen/extracellular signal-regulated kinase inhibitors. Retin Cases Brief Rep 12: 172-176, 2018

15. Spaide RF, Noble K, Morgan A and Freund KB: Vitelliform macular dystrophy. Ophthalmology 113: 1392-1400, 2006.

16. Lima de Carvalho JR Jr, Paavo M, Chen L, Chiang J, Tsang SH and Sparrow JR: Multimodal imaging in best vitelliform macular dystrophy. Invest Ophthalmol Vis Sci 60: 2012-2022, 2019.

17. Querques G, Bux AV, Prato R, Iaculli C, Souied EH and Noci ND: Correlation of visual function impairment and optical coherence tomography findings in patients with adult-onset foveomacular vitelliform macular dystrophy. Am J Ophthalmol 146: 135-142, 2008 .
18. Bitner H, Schatz P, Mizrahi-Meissonnier L, Sharon D and Rosenberg T: Frequency, genotype, and clinical spectrum of best vitelliform macular dystrophy: Data from a national center in Denmark. Am J Ophthalmol 154: 403-412.e4, 2012.

19. Boon CJ, Klevering BJ, Leroy BP, Hoyng CB, Keunen JE and den Hollander AI: The spectrum of ocular phenotypes caused by mutations in the BEST1 gene. Prog Retin Eye Res 28: 187-205, 2009.

20. Kubota D, Gocho K, Akeo K, Kikuchi S, Sugahara M, Matsumoto CS, Shinoda K, Mizota A, Yamaki K, Takahashi H and Kameya S: Detailed analysis of family with autosomal recessive bestrophinopathy associated with new BEST1 mutation. Doc Ophthalmol 132: 233-243, 2016.

21. Wong RL, Hou P, Choy KW, Chiang SW, Tam PO, Li H, Chan WM, Lam DS, Pang CP and Lai TY: Novel and homozygous BEST1 mutations in Chinese patients with best vitelliform macular dystrophy. Retina 30: 820-827, 2010.

22. Tian L, Sun T, Xu K, Zhang X, Peng X and Li Y: Screening of BEST1 gene in a Chinese cohort with best vitelliform macular dystrophy or autosomal recessive bestrophinopathy. Invest Ophthalmol Vis Sci 58: 3366-3375, 2017.

23. Luo J, Lin M, Guo X, Xiao X, Li J, Hu H, Xiao H, Xu X, Zhong Y, Long S, et al: Novel BEST1 mutations and special clinical characteristics of autosomal recessive bestrophinopathy in Chinese patients. Acta Ophthalmol 97: 247-259, 2019.

24. Milenkovic VM, Rivera A, Horling F and Weber BH: Insertion and topology of normal and mutant bestrophin-1 in the endoplasmic reticulum membrane. J Biol Chem 282: 1313-1321, 2007.

25. Katagiri S, Hayashi T, Ohkuma Y, Sekiryu T, Takeuchi T, Gekka T, Kondo M, Iwata T and Tsuneoka H: Mutation analysis of BEST1 in Japanese patients with Best's vitelliform macular dystrophy. Br J Ophthalmol 99: 1577-1582, 2015.

26. Lee CS, Jun I, Choi SI, Lee JH, Lee MG, Lee SC and Kim EK: A novel BEST1 mutation in autosomal recessive bestrophinopathy. Invest Ophthalmol Vis Sci 56: 8141-8150, 2015.

27. Tian R, Yang G, Wang J and Chen Y: Screening for BEST1 gene mutations in Chinese patients with bestrophinopathy. Mol Vis 20: 1594-1604, 2014

28. Johnson AA, Bachman LA, Gilles BJ, Cross SD, Stelzig KE, Resch ZT, Marmorstein LY, Pulido JS and Marmorstein AD: Autosomal recessive bestrophinopathy is not associated with the loss of bestrophin-1 anion channel function in a patient with a novel BEST1 mutation. Invest Ophthalmol Vis Sci 56: 4619-4630, 2015

29. Hartzell HC, Qu Z, Yu K, Xiao Q and Chien LT: Molecular physiology of bestrophins: Multifunctional membrane proteins linked to best disease and other retinopathies. Physiol Rev 88: 639-672, 2008

30. Tsunenari T, Sun H, Williams J, Cahill H, Smallwood P, Yau KW and Nathans J: Structure-function analysis of the bestrophin family of anion channels. J Biol Chem 278: 41114-41125, 2003.

31. Krämer F, Mohr N, Kellner U, Rudolph G and Weber BH: Ten novel mutations in VMD2 associated with best macular dystrophy (BMD). Hum Mutat 22: 418, 2003.

32. Petrukhin K, Koisti MJ, Bakall B, Li W, Xie G, Marknell T, Sandgren O, Forsman K, Holmgren G, Andreasson S, et al: Identification of the gene responsible for best macular dystrophy. Nat Genet 19: 241-247, 1998.

33. Ergun E, Costa D, Slakter J, Yannuzzi LA and Stur M: Photodynamic therapy and vitelliform lesions. Retina 24: 399-406, 2004

34. Guziewicz KE, Zangerl B, Komaromy AM, Iwabe S, Chiodo VA, Boye SL, Hauswirth WW, Beltran WA and Aguirre GD: Recombinant AAV-mediated BEST1 transfer to the retinal pigment epithelium: Analysis of serotype-dependent retinal effects. PLoS One 8: e75666, 2013.

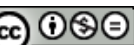

This work is licensed under a Creative Commons Attribution-NonCommercial-NoDerivatives 4.0 International (CC BY-NC-ND 4.0) License. 